\title{
A life in the day of a duty consultant
}

\section{G Hughes}

ts $5.00 \mathrm{am}$

The metallic trill of your mobile phone wakes you, sounding like clanging dustbin lids. You never sleep well when you are on call; the subliminal anxiety of awaiting a call constantly nibbles away, never letting you relax properly. The department is overloaded with patients. The 4 hour target is the stuff of legend. The staff need your help. Perversely, it's almost a relief to get called in; the unbearable lightness of being available is answered.

Feeling guilty to take a shower, you grab something to drink and drive in. The roads are quiet, but then it is Sunday morning. As you walk into the hospital you can see the waiting room is very crowded. The smiles from your medical, nursing and clerical colleagues reflect their relief that a member of the cavalry has arrived. You exchange greetings and ask a few pertinent questions about the volume and acuity of the night's work. There are no surprises. You go to your office, grab a pen and your stethoscope metaphorically if not physically roll up your sleeves, take a big sigh and you're off. You work steadily after an initial blitz; deal with some clinical crises, some trivia and all the stuff in between. You keep going. Is this queue never going to end? You drink coffee from time to time, but it's always lukewarm and you never finish a mug. Before you know it the whole day has flown by in a blur and its 8.30 in the evening. You've had enough. This is the time your scheduled day shift is due to end anyway. You go home exhausted and hungry. You realise you've not eaten all day. At home you have your belated breakfast, shower and fall asleep on the sofa.

Its $11.00 \mathrm{pm}$.

The metallic trill of your mobile phone wakes you, sounding like clanging dustbin lids. What day is it? Oh-it's still Sunday. There are multiple casualties from an RTA. You are needed both as a pair of hands and as a trauma team leader. You drive in, feeling slightly unsafe to do so. You also feel slightly unsafe to lead a trauma team. You have to though, as there is no backup.

As you deal with the fallout from the accident, having just spoken to the police and some very distressed relatives, you notice with quiet despair that one of the junior doctors in your department leaves at $1.00 \mathrm{am}$ on the dot, as her shift ends. She makes no acknowledgment of the department's or your predicament. Her shift has ended and she is leaving. You check the waiting room, finally wrap up what needs to be wrapped up and head home at 3.00 am. It's summer. The morning chorus is a pleasant backdrop as you fall asleep at home.

When you wake later that day, no longer on call, you wonder why you feel washed out. Then you remember. You reflect on the previous day. Were you safe? Did you make the right decisions? Did you say the right thing to the relatives? Were you grumpy and off hand or were you courteous and professional? You cannot remember. You later go for a run.

Over the ensuing days you cannot get some niggles out of your mind. There is something wrong but it's unclear exactly what. You know that you have signed the new consultant contract and you know that you have agreed your working patterns with the chief executive of your trust and with the excellent colleagues in your department, but was it meant to be like this? OK, you had the Friday off before the weekend, but you still had to go in to the department to do some "paperwork" and go to a couple of meetings. You know that days like the one you've just experienced only happen occasionally; they are the exceptions that prove the rule, whatever that means.

You think about the modern working conditions for junior doctors. The European Working Times Directive is clear and prescriptive, but does it apply to consultants? You reflect that something seems to have been lost, but you cannot quite put your finger on it; then it comes to you; flexibility, and true camaraderie. Organisational loyalty? Professionalism? Do junior doctors these days only work to the letter of their contract and that's it? Are consultants the fall guys who will always have to fill the gaps? You curse yourself because you sound like an old fart. You inwardly groan with embarrassment to hear yourself thinking the cliche you heard in your own junior days; "in my day we had to.........", "you don't know what hard work is........". You sound like a sun-dried, retired Colonel Blimp from Bagshot. You laugh at yourself and concede with grace that it is a generational thing and there is little you can do about it.

What concerns you is that the few times a year when you have to work with such intensity you feel you become clinically unsafe. Are you a risk for the patient and ultimately for yourself? Your ability to handle days like this will weaken as you age, it has to be said. Are you whinging and self-pitying or do you have a genuine gripe?

Is it possible to rethink how we provide an on-call service and support the department in times of severe stress? Can we limit the number of hours of consistent high-pressure work? Can we have a second on-call? Can we have day and night cover? Should I just accept it? It's all quite complex and you don't know the solution. Surely you are not the only person thinking like this.

Then you have an idea. You contact the EMJ to ask if they will consider publishing an editorial on the topic. The journal agrees to do it, anonymising the source.

Emerg Med J 2006;23:892.

doi: $10.1136 / \mathrm{emj} .2006 .042812$

Correspondence to: Geoffrey Hughes, The Emergency Department, Royal Adelaide Hospital, North Terrace, Adelaide 5000, Australia; cchdhb@yahoo.com

Accepted 10 October 2006

Competing interests: None declared. 